\title{
Nonsmall Cell Lung Cancer Therapy: Insight into Multitargeted Small-Molecule Growth Factor Receptor Inhibitors
}

\author{
Mridul Roy, ${ }^{1}$ Yu-Hao Luo, ${ }^{1}$ Mao Ye, ${ }^{2}$ and Jing Liu ${ }^{1}$ \\ ${ }^{1}$ Molecular Biology Research Center, School of Life Science and State Key Laboratory of Medical Genetics of China, \\ Central South University, Changsha, Hunan 410078, China \\ ${ }^{2}$ Molecular Science and Biomedicine Laboratory, State Key Laboratory for Chemo/Biosensing and Chemometrics, College of Biology, \\ College of Chemistry and Chemical Engineering, Hunan University, Changsha 410082, China
}

Correspondence should be addressed to Mao Ye; yemaocsu@hotmail.com and Jing Liu; jingliucsu@hotmail.com

Received 14 April 2013; Accepted 13 June 2013

Academic Editor: Beric Henderson

Copyright ( 2013 Mridul Roy et al. This is an open access article distributed under the Creative Commons Attribution License, which permits unrestricted use, distribution, and reproduction in any medium, provided the original work is properly cited.

To date, lung cancer is the leading cause of cancer-related death worldwide, among which nonsmall cell lung cancer (NSCLC) comprises about $85 \%$. Taking into account the side effects of surgery, radiation, platinum-based doublet chemotherapy, and the growth self-sufficiency characteristic of cancer cells, drugs have been discovered toward growth factor receptor (GFR) to treat NSCLC. As expected, these drugs provide a greater benefit. To increase the efficacy of such growth factor receptor tyrosine kinase inhibitors (RTKIs), coinhibition of GFR signaling pathways and combination of inhibitors along with radiation or chemotherapy have drew intense insight. Although clinical trials about single-agent RTKIs or their combination strategies suggest their increase potency against cancer, they are not beyond adverse effects, and sometimes the effects are more deadly than chemotherapy. Nevertheless the hope for RTKIs may be proved true by further researches and digging deep into cancer therapeutics.

\section{Introduction}

Worldwide, the impact of lung cancer is enormous making it the leading cause of cancer-related mortality [1]. Nonsmall cell lung cancer (NSCLC) accounts for approximately 85\% of all cases of lung cancer [2]. Standard first-line treatment options for NSCLC depend on disease and patient characteristics and may include surgery, platinum-based doublet chemotherapy, and targeted therapies [3]. Although surgical resection is curative if diagnosis occurs at early stage I or stage II disease, almost half of all newly diagnosed patients are with advanced-stage disease and candidates for palliative systemic therapies. Chemotherapy for nonsmall cell lung cancer (NSCLC) has shown modest improvements in patients with stages II and IIIA NSCLC with an improvement in both overall survival (OS) and quality of life $[4,5]$. This first-line, platinum-based doublet chemotherapy regimen is associated with modest clinical benefits, but it has significant toxicities [6]. Furthermore chemotherapy combinations for more advanced disease have shown to convey no benefit on overall survival or quality of life beyond $4-6$ cycles $[7,8]$.
As knowledge about molecular abnormalities that drive cell growth and proliferation for lung cancers has grown and as NSCLC currently has a 5-year survival rate of less than $20 \%$ [9], there is clearly a need for the development of more effective therapies. According to Hanahan and Weinberg [10], the cell surface receptors that transduce signals into the cell are the targets of deregulation during tumor progression resulting in self-sufficiency in growth signal, one of the major hallmarks for cancer cells. Growth factor receptors (GFRs) are overexpressed in many cancers which may enable the cancer cell to become hyperresponsive to ambient level of growth factors and even ligand-independent signaling. This observation provides the rationale for the interest of research to develop anti-GFR compounds.

Although treatments, which target individual pathway, have showed clinical successes, the ability of tumor cell to develop resistance to circumvent inhibition of a single signaling pathway drives the urge to target multiple signaling pathways. Therapeutic approaches to inhibit multiple pathways using multiple single-targeted agents may help to maximize the suppression of oncogenic processes involved 


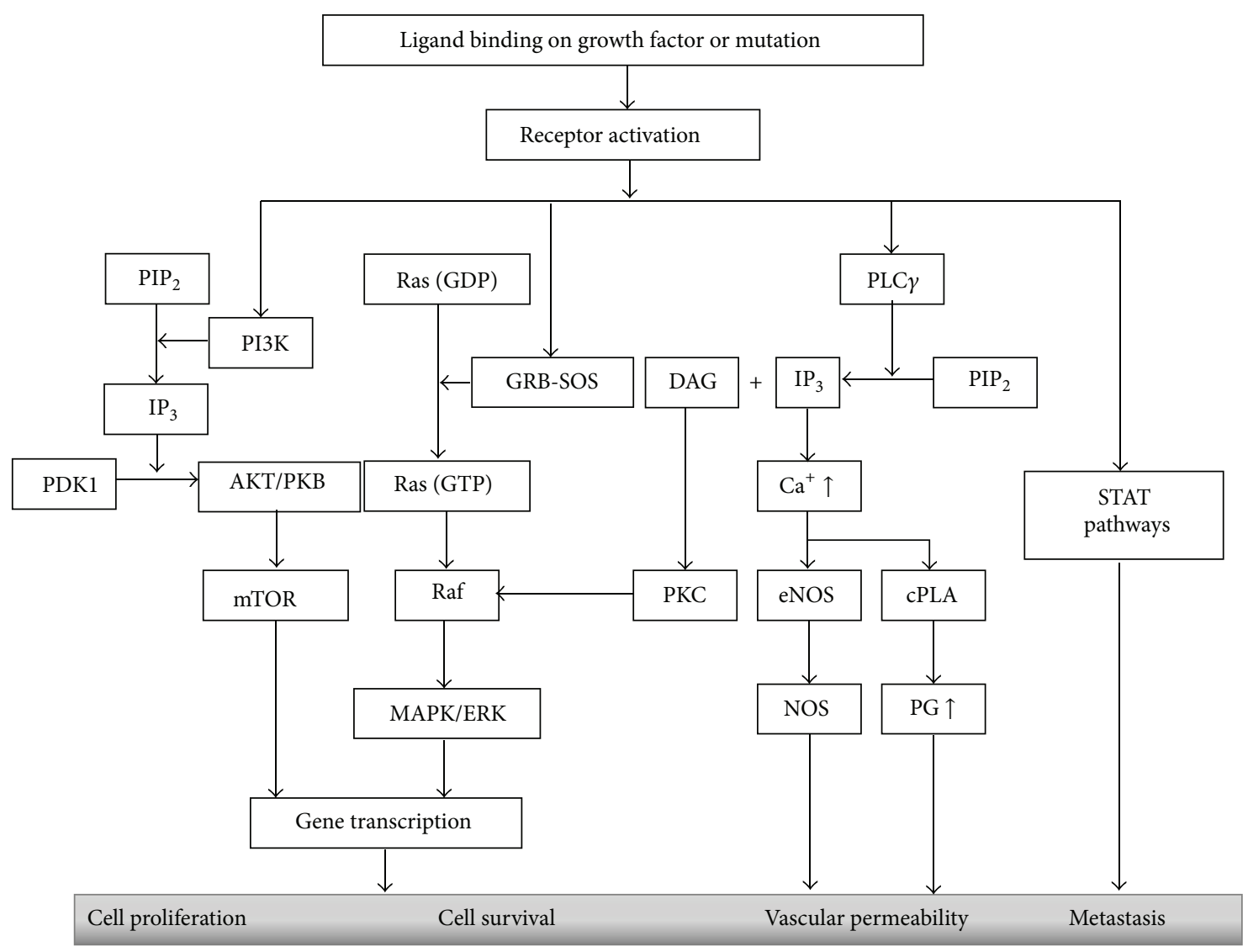

FIgure 1: Possible pathways for growth factor mediated tumorigenesis. Activation of receptor can lead to cell proliferation, survival, angiogenesis, and metastasis via four possible pathways; the phosphatidylinositol 3-kinase (PI3K)/Akt/mammalian target of rapamycin (mTOR) pathway, Ras/Raf/mitogen-activated protein kinase (MAPK) pathway, nitric oxide synthase (NOS), and prostaglandin (PG) involving phospholipase $\mathrm{C} \gamma(\mathrm{PLC} \gamma)$ /protein kinase $\mathrm{C}$ (PKC) pathway and signal transducer and activator of transcription (STAT) pathway.

in disease progression. Using a single multitargeted agent, rather than to use multiple single agents, to individually attack multiple targets is an alternative strategy. In this study we reviewed the mechanisms that lung cancer cells carry out for growth, proliferation, angiogenesis, and metastasis by using GFRs, and how they are being cotargeted by smallmolecule inhibitors and current trial use of these molecules as treatment of NSCLC at I/II/III phases. In addition we reviewed the studies about the combination of targeting agents with other molecules to achieve a better efficacy.

\section{Role of GFRs on NSCLC}

The growth factor receptors respond to their specific ligands and mediate tumorigenic activity through variety of signaling pathways (Figure 1). In case of NSCLC it was shown that epidermal growth factor receptor (EGFR) is overexpressed and specific somatic mutations occurred in their intracellular domain which may influence prognosis and significantly related to stage, survival, and chemotherapy response [11]. There are four main intracellular signaling pathways involved in the activation of EGFR: Ras/mitogenactivated protein kinase (MAPK), phosphatidylinositol 3kinase (PI3K)/Akt, phospholipase $\mathrm{C} \gamma(\mathrm{PLC} \gamma)$, protein kinase
$\mathrm{C}$ (PKC), and signal transducer and activator of transcription (STAT) [12]. Like EGFR, vascular endothelial growth factor (VEGF) also plays a critical role in tumorigenesis, and these two pathways are linked together [13]. VEGF is associated with neovascularization and influences progression of NSCLC [14], and inhibition of VEGF or its receptor (VEGFR) correlate with impaired cell-cycle distribution, overall survival, and chemotherapy response $[15,16]$. VEGFR mediates its angiogenic effect through PI3K/PLC $\gamma$ pathway [17]. Enhanced activity of platelet derived growth factor (PDGF) is associated with tumorigenesis and angiogenesis [18] and inhibition of PDGF receptor (PDGFR) impede tumor growth by impairing periendothelial cell recruitment in A549 NSCLC cell line [19]. Coexpression of PDGF-B and VEGFR-3 is associated with poor survival in NSCLC patients [20]. The fibroblast growth factor (FGF) family comprises 22 ligands [21] with numerous isoforms of FGF receptor tyrosine kinases generated by messenger RNA splicing from 4 genes (FGFR1, FGFR2, FGFR3, and FGFR4) [22]. As reviewed by Semrad and Mack [23], FGFs and FGFRs play multiple potential mechanisms for tumor proliferation, survival, neoangiogenesis, and metastases in NSCLC. Signaling through FGFRs is mediated by PI3K/PLC $\gamma$ and Ras/mitogenactivated protein kinase (MAPK) signaling cascades [23]. 
TABLE 1: GFR targeted monoclonal antibodies (mAbs) for NSCLC therapy.

\begin{tabular}{|c|c|c|c|c|}
\hline mAb name & Targeted GFR & Source & Current developed phase & Ongoing trial $^{\mathrm{a}}$ \\
\hline Cetuximab & EGFR & Chimeric & III & $\begin{array}{l}\text { NCT00408499 } \\
\text { NCT00397384 } \\
\text { NCT00946712 } \\
\text { NCT00533949 } \\
\text { NCT00368992 } \\
\text { NCT01059188 } \\
\text { NCT00867009 } \\
\text { NCT00842712 }\end{array}$ \\
\hline Cixutumumab & IGF-1R & Human & $\mathrm{I} / \mathrm{II}$ & $\begin{array}{l}\text { NCT00955305 } \\
\text { NCT01232452 } \\
\text { NCT01263782 } \\
\text { NCT00887159 }\end{array}$ \\
\hline EMD 72000 (Matuzumab) & EGFR & Humanized from mouse & II & - \\
\hline Figitumumab (CP-751,871) & IGF-1R & Human & II & NCT00728390 \\
\hline Ganitumab (AMG 479) & IGF-1R & Human & I & NCT01327612 \\
\hline Necitumumab (IMC-11F8) & EGRF & Human & III & $\begin{array}{c}\text { NCT00982111 } \\
\text { NCT00981058 } \\
\text { NCT01769391 } \\
\end{array}$ \\
\hline Nimotuzumab & EGFR & Humanized from mouse & II & $\begin{array}{l}\text { NCT01498562 } \\
\text { NCT01393080 } \\
\end{array}$ \\
\hline Olaratumab (IMC-3G3/LY3012207) & PDGFR- $\alpha$ & Human & II & NCT00918203 \\
\hline Panitumumab & EGFR & Human & $\mathrm{I}$ & $\begin{array}{l}\text { NCT00979212 } \\
\text { NCT01042288 } \\
\text { NCT01038037 }\end{array}$ \\
\hline Ramucirumab (IMC-1121B) & VEGF-2 & Human & III & $\begin{array}{l}\text { NCT01160744 } \\
\text { NCT01168973 } \\
\text { NCT01703091 }\end{array}$ \\
\hline Trastuzumab & HER-2 & Humanized from mouse & II & $\begin{array}{l}\text { NCT00847366 } \\
\text { NCT01148849 }\end{array}$ \\
\hline
\end{tabular}

http://www.clinicaltrial.gov/, access date March 16, 2013.

The insulin-like growth factor (IGF) pathway is linked to the deregulation of cell growth and metastasis, and its growthpromoting activities are mediated by the IGF-1R [22]. IGF$1 \mathrm{R}$ expression is associated with reduced disease-free survival (DFS) in NSCLC [24]. IGF-1R is a glycoprotein composed of two extracellular alpha subunits that preferentially bind IGF-1 and IGF-2 and insulin with a lesser affinity. The two intracellular beta subunits contain the tyrosine kinase domain responsible for activating the IRS/PI3K/AKT/mTOR pathway and the Ras/Raf/MAPK pathways that promote cell growth, transformation, migration, and survival $[25,26]$.

\section{GFR Targeted Therapy for NSCLC}

Downstream signaling of GFRs can be abrogated by (1) inhibition of receptor expression using gene therapy (antisense approach), (2) antagonistic monoclonal antibodies $(\mathrm{mAb})$ that prevent binding of ligands to receptors, or (3) pharmacologic (low-molecular weight) receptor-selective tyrosine kinase inhibitors (RTKIs). MAb and TKI actions are the preferred approaches currently under intense clinical development [27].
3.1. Mechanism of Action of mAbs. In the early $1980 \mathrm{~s} \mathrm{mAb}$ was first applied for cancer treatment, about 10 years earlier than the development of small-molecule TKIs. Although early clinical trials with murine $\mathrm{mAbs}$ failed owing to their short half-life and limited activity, genetic engineering has made it possible to design chimeric mouse-human mAbs and humanized mAbs [28]. Among a number of these antibodies (Table 1), bevacizumab, a humanized $m A b$ to vascular endothelial growth factor (VEGF), is currently approved for use in combination with chemotherapy in multiple countries for the treatment of patients with metastatic NSCLC [29]. The mAbs can confer their antitumor activity by two putative mechanisms. For the direct action, it can block the function of target signaling molecules resulting in inhibition of cellcycle progression, regression of angiogenesis, induction of apoptosis, and internalization of receptors or it can be conjugated with toxins, cytokines, and even with small-molecule agents $[28,30]$. The second or indirect mechanism of $\mathrm{mAb}$ is mediated by the immune system through complementdependent cytotoxicity (CDC), complement-dependent cellmediated cytotoxicity (CDCC), or antibody-dependent cellular cytotoxicity (ADCC) [31] (Figure 2(a)). 
3.2. Mechanism of Action of RTKIs. Most small-molecule kinase inhibitors, discovered to date, are ATP mimetic that compete with ATP for the ATP-binding cleft of the kinase domain of the receptor. The binding may differ for the inhibitors, and at least three types of binding have been found. Among them the first is recognition of the active conformation of a kinase by the inhibitor and mimic the hydrogen bond that is normally formed by ATP [32]. These inhibitors, for example, sunitinib, usually present one to three hydrogen bonds to the ATP-binding site [32, 33]. The second type of inhibitors modulates kinase activity and its downstream signaling in an allosteric way by using a hydrophobic pocket to (indirectly) compete with ATP [34]. Sorafenib belongs to this kind of kinase inhibitors group [35]. The third kind is covalent inhibitors that react with electron rich sulfur (S) atom of cysteine residue by sharing electrons at specific sites of the kinase which allows the inhibitor to block binding of ATP to the kinase and prevents its activation [36]. Example of this kind of inhibitor includes vandetanib [37] (Figure 2(b)).

It is quite impossible to predict which of these two approaches can be more effective; moreover there is no clear difference in the spectrum of cancers targeted by mAbs and small-molecule inhibitors. One advantage of TKIs over mAbs is that they are administered orally, making them ideal for chronic maintenance therapy although they have a shorter half-life than mAbs. Furthermore these antibodies induce downregulation of surface receptors in addition to blocking ligand binding to receptors as well as incite antibodydependent cell-mediated cytotoxicity [38].

\section{Inhibition of Multiple Signalling by RTKIs}

\subsection{VEGFR/PDGFR Dual Inhibition}

4.1.1. Sorafenib. Sorafenib (Bay 43-9006, Nexavar, Bayer; Leverkusen, Germany) can block the phosphorylation of VEGFR, PDGFR, Raf, and stem cell factor receptor (KIT). In phase II studies, single-agent sorafenib prolonged progression-free survival (PFS) [39]. Several studies about combination of sorafenib with chemotherapeutic agents reported no beneficial effects. Placebo-controlled phase III trials of carboplatin/paclitaxel [40] and gemcitabine/cisplatin [41,42] alone or with sorafenib have demonstrated no additional benefit of response rate, PFS, or OS. Patients with squamous histology were found to be more vulnerable in mortality index. But the combination of sorafenib with another EGFR tyrosine kinase inhibitor erlotinib proved promising clinical activity in patients with advanced NSCLC with an acceptable safety profile. A multicenter phase II study of erlotinib and sorafenib in patients with adenocarcinoma (72\%), squamous (10\%), large cell (12\%), and NSCLC NOS (6\%) histology reported an OS of 10.9 months with its adverse events which included fatigue (16\%), hand-foot skin reaction (16\%), rash (16\%), diarrhea (14\%), and hypophosphatemia (42\%) [43]. Another phase II study (patients with squamous histology were excluded) demonstrated the higher activity of sorafenib/erlotinib combination than sorafenib/gemcitabine combination with

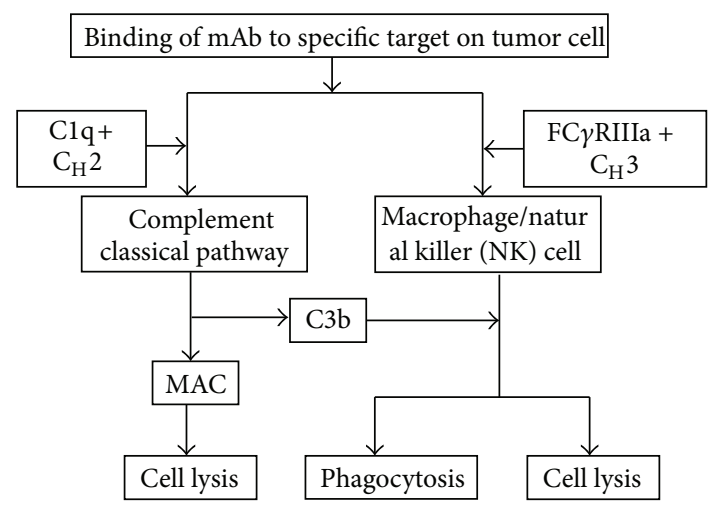

(a)

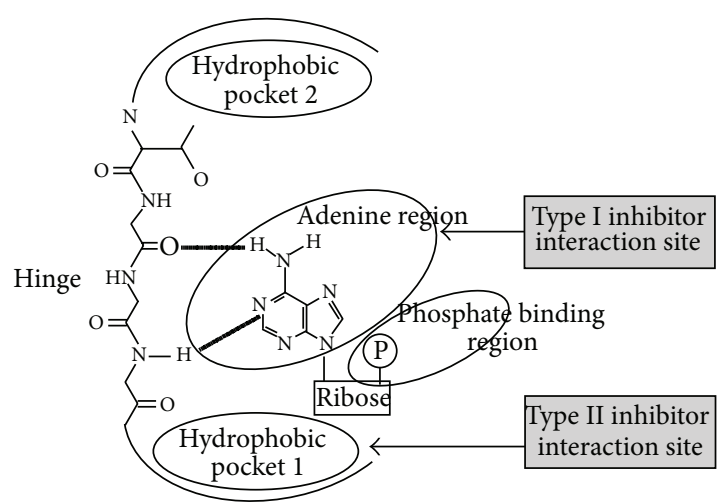

(b)

FIGURE 2: Growth factor receptor inhibition. (a) Immunomodulatory mechanism for monoclonal antibodies. Binding of monoclonal antibodies (mAbs) to a specific target on a tumour cell can cause either complement-dependent cytotoxicity (CDC) by the interaction of Clq complement factor with the $\mathrm{C}_{\mathrm{H}} 2$ constant region of the $\mathrm{mAb}$, which leads to the activation of complement classical pathway and induces the formation of a membrane-attack complex (MAC) for the lysis of tumour cells or antibody-dependent cellular cytotoxicity (ADCC) by the interaction of $\mathrm{C}_{\mathrm{H}} 3$ region of the mAbs with Fc $\gamma$ RIIIa expressed by effector cells (macrophages or NK cells) which leads to phagocytosis by macrophages or undergo cytolysis by NK cells. C3b, which is generated during CDC, can facilitate phagocytosis and cytolysis through its interaction with macrophage or natural killer (NK) cell. This effect is termed as complement-dependent cell-mediated cytotoxicity (CDCC). (b) Small-molecule receptor tyrosine kinase inhibitor interaction to its specific site. Small-molecule tyrosine kinase inhibitors (TKIs) function as ATP analogues to compete with ATP for their binding site and block the receptor mediated downstream signaling.

a higher 1-year survival rate for first group patients [44]. Benefit for the combination of erlotinib/sorafenib over single-agent erlotinib with respect to PFS (3.38 months for sorafenib/erlotinib versus 1.94 months for placebo/erlotinib) and OS (8 months for sorafenib/erlotinib versus 4.5 months for placebo/erlotinib) has also been reported [45]. According to Adjei et al. [46], in patients with advanced NSCLC who achieve a prolonged response or stable disease with sorafenib given as a single agent or as part of a combination regimen, 
sorafenib treatment could be continued until disease progression without major long-term safety or tolerability problems. These studies can conclude that combination of erlotinib and sorafenib would take NSCLC therapy into a plateau.

4.1.2. Sunitinib. Sunitinib (SU11248, Sutent, Pfizer; New London, CT, USA) is an oral, small-molecule, and multitargeted receptor tyrosine kinase (RTK) inhibitor of VEGFR-2 (Flk-1), PDGFR- $\beta$, and KIT, rearranged during transfection (RET), colony-stimulating factor 1 receptor (CSF-1R), and FMSlike tyrosine kinase-3 receptor (FLT3) [47]. Phase II studies of sunitinib on a continuous daily dosing (CDD) schedule suggested sunitinib as a safe and potential therapeutic agent for patients with previously treated advanced NSCLC of all histologies, though single-agent sunitinib was associated with a few manageable hematologic and neurologic abnormalities [48, 49]. Combinations of sunitinib with other agents were studied in order to gain high efficacy. But the results were not so satisfactory because of toxicities. A phase III trial revealed sunitinib plus erlotinib did not improve OS compared with erlotinib alone (9.0 months versus 8.5 months) in patients with NSCLC of nonsquamous (about $82 \%$ adenocarcinoma) histology, but the combination was associated with a statistically significant longer PFS (3.6 months versus 2.0 months) and greater overall response rate (ORR) (10.6\% versus 6.9\%) [50]. That trial also revealed a greater grade 3 or higher toxicities with combined therapy. Earlier a study about sunitinib with bevacizumab plus paclitaxel/carboplatin was discontinued because of toxicities including neutropenia, thrombocytopenia, leukopenia, and fatigue on its first phase [51]. However Blumenschein Jr. et al. [52] suggested on their randomized, double-blind, and multicenter study that a dosage of sunitinib concurrently with erlotinib was tolerable in subjects with platinum-refractory NSCLC of adenocarcinoma (70.0\%), squamous cell (26.7\%), and large cell (3.3\%) histology, but the adverse effects were not totally removed with diarrhea $(76.9 \%)$, fatigue $(61.5 \%)$, and decreased appetite (53.8\%) being the most frequent adverse events.

4.1.3. Cediranib. Cediranib (AZD2171, Recentin, AstraZeneca; Wilmington, DE, USA) is an oral TKI that targets VEGFR-1, VEGFR-2, VEGFR-3, PDGFRs, and c-kit [53]. Cediranib as a monotherapy was generally well tolerated and showed encouraging antitumor activity in a phase I, dose escalation, and pharmacokinetic study in patients with advanced solid tumor [54]. Phase I data suggested that cediranib could be combined with standard doses of cisplatin and gemcitabine in patients with advanced NSCLC with promising activity but predictable and manageable toxicity [55]. Based on these data, a randomized phase II/III trial of carboplatin/paclitaxel plus cediranib or placebo as first-line therapy for advanced NSCLC was initiated [56]. Although PFS favored the combination of chemotherapy plus cediranib regardless of patient's histology, the study encountered excessive toxicities including hypertension, hypothyroidism, hand-foot syndrome, hypoalbuminemia, and an imbalance in treatment related deaths (10 and 2 for chemotherapy plus cediranib and chemotherapy plus placebo, resp.). A randomized phase II study of gemcitabine and carboplatin with or without cediranib as first-line therapy in advanced NSCLC of squamous (15.5\% versus $27.6 \%)$, adenocarcinoma $(37.9 \%$ versus $55.2 \%$ ), and other (46.6\% versus $17.2 \%$ ) histologies did not meet its primary endpoint of overall response rate (ORR) (19\% versus 20\%) but met its secondary endpoint of 6-month progression-free survival (48\% versus protocolspecified threshold of at least $40 \%$ ) with more grade 3 plus nonhematologic adverse events experienced by cediranib group [57].

4.1.4. Linifanib. Linifanib (ABT-869, Abbott; Abbott Park, IL, USA) targets VEGFR-1, VEGFR-2, VEGFR-3, and PDGFR [58]. Phase I study showed that linifanib had a favorable safety profile with minimal dose limiting toxicities (DLTs) and median PFS of 5.7 months [59]. Another phase II trial of linifanib in 139 (122 nonsquamous and 17 squamous histology) patients with advanced nonsmall cell lung cancer showed that it was active in advanced nonsmall cell lung cancer as second- or third-line therapy. The study also reported a median PSF of 3.6 months, median OS of 9.0 months with the adverse events, comprising fatigue (42\%), decreased appetite (38\%), hypertension (37\%), diarrhea $(32 \%)$, nausea $(27 \%)$, palmar-plantar erythrodysesthesia (24\%), and proteinuria (22\%) [60]. Comparatively low dose linifanib can be a potent small-molecule inhibitor against NSCLC though study about its combination with other targeting agents is limited.

4.1.5. Axitinib. Axitinib (AG-013736, Pfizer; New London, CT, USA) is an oral TKI with activity against VEGFR, PDGFR, and c-kit [61]. In vitro, axitinib enhances efficacy of chemotherapeutic drugs (topotecan and mitoxantrone) via inhibiting the drug transport function of adenosine triphosphate- (ATP-) binding cassette subfamily G member 2 (ABCG2) and reversed ABCG2-mediated multidrug resistance (MDR) [62]. In a phase I trial study Kozloff et al. [63] demonstrated that administration of axitinib with paclitaxel/carboplatin or gemcitabine/cisplatin regimens was well tolerated with improved clinical efficacy. They have also reported no grade $\geq 3$ haemoptysis among 12 patients with squamous cell NSCLC out of total 49 patients suggesting clinically working further with this drug.

4.1.6. Motesanib. Motesanib (AMG 706; Amgen, Thousand Oaks, CA, USA) is an orally administered potent small molecule, which targets VEGFR-1, VEGFR-2, VEGFR-3, PDGFR, and KIT [64]. In human NSCLC xenograft models, motesanib has been proved as a potent antitumor agent and that it had enhanced activity when combined with cisplatin or docetaxel [65]. But clinical trials of combination have different results. Several phase III trials have failed to achieve their primary endpoint of OS. A randomized, double-blind phase III study of motesanib plus carboplatin/paclitaxel in 1090 patients (of those, 890 had adenocarcinoma) with advanced NSCLC demonstrated the combination did not significantly improve OS over carboplatin/paclitaxel alone, with a median OS of 13 months and 11 months and median PFS of 5.6 months 
and 5.4 months for carboplatin/paclitaxel plus motesanib and carboplatin/paclitaxel alone, respectively [50]. The grade $\geq 3$ and grade 5 adverse effects were higher with motesanib treatment. Similarly another MONET1 (Motesanib NSCLC Efficacy and Tolerability) study reported improvement on PFS but no OS benefit with increased toxicity [66].

\subsection{VEGFR/EGFR Dual Inhibition}

4.2.1. Vandetanib. Vandetanib (AZD6474, Zactima, AstraZeneca; Wilmington, DE, USA) is a multitargeted tyrosine kinase inhibitor (TKI) that inhibits VEGFR, EGFR, and RET [67]. Single-agent vandetanib has antitumor activity with an acceptable safety and tolerability profile [68]. Combinations of vandetanib with chemotherapeutic agents are being evaluating for better efficacy as expected combination of vandetanib with paclitaxel and/or carboplatin have increased PFS [69]. But the adverse effects of combining therapy are still in concern. Three phase III studies also provide contradictory concepts. The ZEAL trial $(N=534)$, which compared vandetanib plus pemetrexed versus placebo plus pemetrexed, failed to meet its primary endpoint of prolonged PFS [70]. Whereas the ZODIAC trial, which compared vandetanib plus docetaxel ( $N=694$; of those 412 had adenocarcinoma, 184 had squamous, and 98 had other histology) versus placebo plus docetaxel (697 patients; of those 417 had adenocarcinoma, 160 had squamous, and 120 had other histologies) [71], the ZEPHYR trial (924 patients; of those 726 had adenocarcinoma, 102 had squamous, and 96 had other histologies) evaluated vandetanib in patients with advanced NSCLC, and prior EGFR TKI therapy demonstrated significantly improved PFS for the combination but did not significantly improve OS [72]. In addition both studies showed higher incidence of adverse effects for vandetanib group.

4.2.2. XL647. XL647 (EXEL 7647) is an oral TKI with activity against EGFR, HER2, VEGFR, and EphB4 [73]. XL647 inhibits EGFR phosphorylation as well as phosphorylation of downstream signals (AKT, ERK). XL647 demonstrated antitumor activity in patients with EGFR-activating mutations and adenocarcinoma histology (41 patients) in a phase II study with most common adverse effects being diarrhea, nausea, and fatigue [74]. XL647 has also showed to be active against cells containing the T790M mutation, which have been associated with emerging resistance to first-generation EGFR TKIs such as gefitinib or erlotinib [75]. But in a phase II study of 41 patients among those 38 patients was with adenocarcinoma, 1 with squamous and 2 with undefined histology; it has failed to meet the primary endpoint of response rate in patients with T790M with significantly worse progressionfree survival [76]. Further studies are required to develop this agent as a potential second-generation TKI to delay or overcome acquired resistance to first-generation EGFR TKIs.

\subsection{VEGFR/FGFR Dual Inhibition}

4.3.1. Brivanib. Brivanib (BMS-540215, Bristol-Myers Squibb; New York, NY, USA) is a small-molecule TKI, formulated as an orally administered l-alanine ester prodrug, brivanib alaninate [77]. It is the first-selective dual inhibitor of FGF and VEGF signaling [78], and preclinical studies have demonstrated that dual inhibition of FGF and VEGF signaling by brivanib has strong antiangiogenic effects [79]. In phase I study in patients with advanced or metastatic solid tumors, brivanib demonstrated promising antiangiogenic and antitumor activity and manageable toxicity with most frequent serious toxic effects recorded being nausea, pyrexia, aspartate aminotransferase (AST) or alanine aminotransferase (ALT) elevations, and thrombocytopenia [80, 81]. Synergistic activity of ixabepilone plus brivanib has suggested a promising anticancer activity both in vitro and in vivo [82].

\subsection{VEGFR/PDGFR/FGFR Triple-Target Inhibition}

4.4.1. Nintedanib. Nintedanib or BIBF 1120 (Boehringer Ingelheim; Ingelheim, Germany) is an oral small-molecule triple kinase inhibitor which inhibits VEGFR, PDGFR, and FGFRs and members of the Src family and flt-3 [83]. Singleagent treatment of nintedanib (BIBF 1120) in advanced NCSLC was well tolerated, with median PFS of 6.9 weeks and median overall survival (OS) of 21.9 weeks [84]. 73 patients (40 with adenocarcinoma, 9 with large cell carcinoma, 17 with squamous cell carcinoma, and 7 undefined histology) were enrolled, and, according to this phase II double-blind study, drug-related adverse effects were nausea (57.5\%), diarrhoea (47.9\%), vomiting (42.5\%), anorexia (28.8\%), abdominal pain $(13.7 \%)$, and reversible elevation of alanine transaminase (13.7\%) and aspartate aminotransferase (9.6\%). This drug also has proved its safety and well tolerability on its phase I trials in combination with chemotherapy. Phase I trial of 26 patients (7 with adenocarcinoma, 3 with squamous, 1 with large cell carcinoma, 1 with adenosquamous, and 14 with unspecified histology) with recurrent advanced-stage NSCLC receiving BIBF 1120 in combination with standard-dose pemetrexed was tolerable, with promising signs of efficacy [85]. For combination with paclitaxel and carboplatin, nintedanib demonstrated an acceptable safety profile in a phase I trial [86]. These findings indicate nintedanib as a potential triple kinase inhibitor and as a candidate for further study.

4.4.2. Pazopanib. Pazopanib (GW786034, GlaxoSmithKline; London, UK) is another triple targeted TKI targeting VEGF, PDGFR, and FGFR [87]. Phase II study of pazopanib monotherapy in 35 patients (23 with adenocarcinoma, 4 with squamous, and 8 with other histology) with stage I/II NSCLC demonstrated well tolerability with $86 \%$ patients who achieved tumor volume reduction after pazopanib treatment [88]. Clinical data with pazopanib activity in advanced disease stage and in combination with other drugs are strikingly limited. A phase I study of pazopanib plus gemcitabine combination therapy in patients with advanced solid tumors explored that the combination was tolerable, and there was no apparent pharmacokinetic interaction [89].

\section{Increasing Potential of TKIs}

A rationale for combining molecular targeted therapies might confer additional clinical benefit and eventually reduce 
toxicities compared with standard chemotherapy in patients with advanced disease. Certainly cancer treatment strategies have been blessed with targeted therapy, but, for multitargeted TKIs, failure to respond is more frequent. Searching the reasons behind such failure of multitargeted therapies is a great scientific issue nowaday. Regarding toxicity, it is still a question whether the therapeutic indices of these treatment strategies are sufficiently enhancing. Clinical trials for vandetanib, sorafenib, sunitinib, and cediranib to treat NSCLC were all stopped early due to futility or toxicity issues [40, $42,51,56]$. Resistance occurring from mutation or utilization of cross-talk and redundancy in signaling pathways by the tumor cells remains the rule rather than the exception. But it is not clear whether the failure of multitargeted RTKIs to treat NSCLC arise from resistance as found for the first generation TKIs like gefitinib, erlotinib or crizotinib [90, 91]. Identifying most responsive patients to a specific agent is crucial for the clinical development of targeted agents in patients with NSCLC. Biomarkers, such as EGFR, KRAS, and BRCA1, have been identified to be associated with patient prognosis and/or response to therapy [92]. In fact BATTLE (Biomarker-integrated Approaches of Targeted Therapy for Lung Cancer Elimination) trial for erlotinib, vandetanib, erlotinib plus bexarotene, or sorafenib, based on relevant molecular biomarkers, establishes a new paradigm for individualized treatment approach in lung cancer clinical trials [93]. But it is hard to find studies based on these biomarkers. Studies on population with specific GFR gene mutation are strikingly limited for multitargeted inhibitors. So far, only one phase II study of XL647 has been done on patients with a known sensitizing mutation of EGFR and confirmed antitumor activity of this small-molecule GFR inhibitor [74]. Patient selection irrespective to histology is suspected to be a reason for the failure of multitargeted NSCLC therapy. Histology has been emerged as a potential predictive factor for the outcome of patients with advanced NSCLC treated with EGFR inhibitors [94]. It has been found that, though vast majority of patients have failed to respond to lung cancer therapy, a minority of population, especially those with adenocarcinoma histology and never smokers, showed dramatic tumor reduction with symptomatic improvement $[95,96]$. One of the possible reasons why targeted therapy of lung adenocarcinoma compares favorably with other histologies may be its distinct biological behavior [97]. For example, the expression of HER family members (HER-2), not EGFR expression, is correlated with the efficacy of gefitinib, and it has been found that adenocarcinomas of the lung are more likely to have increased immunohistochemical staining for HER-2 than squamous cell or other NSCLC histologies [96]. Histology should be a key factor for treatment choice in the routine care of patients with NSCLC.

\section{Conclusion}

Nevertheless, to date, a number of platinum-based regimens using as standard therapies have shown modest improvements in both overall survival and quality of life; they are associated with significant toxicities. Moreover our growing knowledge of molecular signaling that makes tumor cell defiant and the poor survival of patients with relapsed or progressive NSCLC reminds us to develop new drugs with better safety profile. Targeting either the VEGFR or EGFR signaling pathways has been clinically validated, and a number of drugs (e.g., bevacizumab, erlotinib, cetuximab, and gefitinib) are currently approved for treating advanced NSCLC. But the ability of solid tumors to develop multiple salvage and resistance pathways that allow them to circumvent inhibition of a single signaling pathway is becoming increasingly evident [98]. Resistance is less likely to arise if multiple regulatory pathways are being targeted at the same time. So, the development of multitargeted TKIs is in concern. Though there is an increasing research interest about these kinds of drugs, their therapy related adverse effects and safety remain in controversy. As we discussed, some clinical trial has been stopped early because of its toxicity issues, and some others could not meet their primary improvement of overall survival; there is still the need to explore more convenient, newer pathways as well as to put insight into coinhibition of existing pathways. It is important to pay more attention to the signaling pathways that are modified by the use of kinase inhibitors. Genomic landscapes for patient-specific kinomes should be provided to appropriately select patients who are most likely to be benefited from RTK-inhibition therapy. The future for growth factor receptor targeted therapy for NSCLC certainly looks bright.

\section{Conflict of Interests}

The authors declare no conflict of interests.

\section{Authors' Contribution}

Mridul Roy and Yu-Hao Luo contributed equally to this work. Mridul Roy and Yu-Hao Luo wrote the paper, Mao Ye and Jing Liu designed and revised the paper.

\section{Acknowledgments}

This work was supported by Grants from the National Natural Science Foundation of China (nos. 81270576, 30971517, 81171950, and 81272220), New Century Excellent Talents in University (NCET-11-0518), Doctoral Fund of Ministry of Education of China (no. 20120162110054), the Fundamental Research Funds for the Central Universities (no. 2011JQ015), and the Innovation Experiment Program for Graduate Students of Central South University (no. 2013zzts268).

\section{References}

[1] American Cancer Society, "Cancer facts and figures 2013," American Cancer Society, Atlanta, Ga, USA, 2013, http://www .cancer.org/acs/groups/content/@epidemiologysurveilance/ documents/document/acspc-036845.pdf.

[2] American Cancer Society, "Non-small cell lung cancer," 2013, http://www.cancer.org/cancer/lungcancer-non-smallcell/ index.

[3] D. S. Ettinger, W. Akerley, H. Borghaei et al., "Non-small cell lung cancer," Journal of the National Comprehensive Cancer Network, vol. 10, no. 10, pp. 1236-1271, 2012. 
[4] F. Fossella, J. R. Pereira, J. von Pawel et al., "Randomized, multinational, phase III study of docetaxel plus platinum combinations versus vinorelbine plus cisplatin for advanced non-small-cell lung cancer: the TAX 326 Study Group," Journal of Clinical Oncology, vol. 21, no. 16, pp. 3016-3024, 2003.

[5] G. V. Scagliotti, P. Parikh, J. von Pawel et al., "Phase III study comparing cisplatin plus gemcitabine with cisplatin plus pemetrexed in chemotherapy-naive patients with advancedstage non-small-cell lung cancer," Journal of Clinical Oncology, vol. 26, no. 21, pp. 3543-3551, 2008.

[6] D. N. Carney, "Lung cancer-time to move on from chemotherapy," New England Journal of Medicine, vol. 346, no. 2, pp. 126128, 2002.

[7] C. von Plessen, B. Bergman, O. Andresen et al., "Palliative chemotherapy beyond three courses conveys no survival or consistent quality-of-life benefits in advanced non-small-cell lung cancer," British Journal of Cancer, vol. 95, no. 8, pp. 966973, 2006.

[8] O. P. Joon, S. W. Kim, S. A. Jin et al., "Phase III trial of two versus four additional cycles in patients who are nonprogressive after two cycles of platinum-based chemotherapy in non-small-cell lung cancer," Journal of Clinical Oncology, vol. 25, no. 33, pp. 5233-5239, 2007.

[9] A. Jemal, M. M. Center, C. DeSantis, and E. M. Ward, "Global patterns of cancer incidence and mortality rates and trends," Cancer Epidemiology Biomarkers and Prevention, vol. 19, no. 8, pp. 1893-1907, 2010.

[10] D. Hanahan and R. A. Weinberg, “The hallmarks of cancer," Cell, vol. 100, no. 1, pp. 57-70, 2000.

[11] M. Fukuoka, S. Yano, G. Giaccone et al., "Multi-institutional randomized phase II trial of gefitinib for previously treated patients with advanced non-small-cell lung cancer (The IDEAL 1 Trial) [corrected]," Journal of Clinical Oncology, vol. 21, no. 12, pp. 2237-2246, 2003.

[12] L. Toschi and F. Cappuzzo, "Understanding the new genetics of responsiveness to epidermal growth factor receptor tyrosine kinase inhibitors," Oncologist, vol. 12, no. 2, pp. 211-220, 2007.

[13] N. A. Pennell and T. J. Lynch Jr., "Combined inhibition of the VEGFR and EGFR signaling pathways in the treatment of NSCLC," Oncologist, vol. 14, no. 4, pp. 399-411, 2009.

[14] G. Fontanini, S. Vignati, L. Boldrini et al., "Vascular endothelial growth factor is associated with neovascularization and influences progression of non-small cell lung carcinoma," Clinical Cancer Research, vol. 3, no. 6, pp. 861-865, 1997.

[15] Y. Wang, L. Huang, Y. Yang, L. Xu, J. Yang, and Y. Wu, "Effects of autocrine vascular endothelial growth factor (VEGF) in nonsmall cell lung cancer cell line A549," Molecular Biology Reports, vol. 40, no. 4, pp. 3093-3099, 2013.

[16] A. Charpidou, I. Gkiozos, M. Konstantinou et al., "Bronchial washing levels of vascular endothelial growth factor receptor-2 (VEGFR2) correlate with overall survival in NSCLC patients," Cancer Letters, vol. 304, no. 2, pp. 144-153, 2011.

[17] N. Ferrara, H. P. Gerber, and J. LeCouter, "The biology of VEGF and its receptors," Nature Medicine, vol. 9, no. 6, pp. 669-676, 2003.

[18] A. Levitzki, "PDGF receptor kinase inhibitors for the treatment of PDGF driven diseases," Cytokine and Growth Factor Reviews, vol. 15, no. 4, pp. 229-235, 2004.

[19] N. Reinmuth, R. Liersch, M. Raedel et al., "Combined antiPDGFRalpha and PDGFRbeta targeting in non-small cell lung cancer," International Journal of Cancer, vol. 124, no. 7, pp. 15351544, 2009.
[20] T. Donnem, S. Al-Saad, K. Al-Shibli, L. T. Busund, and R. M. Bremnes, "Co-expression of PDGF-B and VEGFR-3 strongly correlates with lymph node metastasis and poor survival in non-small-cell lung cancer," Annals of Oncology, vol. 21, no. 2, pp. 223-231, 2010.

[21] A. Beenken and M. Mohammadi, "The FGF family: biology, pathophysiology and therapy," Nature Reviews Drug Discovery, vol. 8, no. 3, pp. 235-253, 2009.

[22] J. M. Knowlden, H. E. Jones, D. Barrow, J. M. W. Gee, R. I. Nicholson, and I. R. Hutcheson, "Insulin receptor substrate-1 involvement in epidermal growth factor receptor and insulinlike growth factor receptor signalling: implication for Gefitinib ('Iressa') response and resistance," Breast Cancer Research and Treatment, vol. 111, no. 1, pp. 79-91, 2008.

[23] T. J. Semrad and P. C. Mack, "Fibroblast growth factor signaling in nonsmall-cell lung cancer," Clinical Lung Cancer, vol. 13, no. 2, pp. 90-95, 2012.

[24] M. Nakagawa, H. Uramoto, S. Oka et al., "Clinical significance of IGF1R expression in nonsmall-cell lung cancer," Clinical Lung Cancer, vol. 13, no. 2, pp. 136-142, 2012.

[25] C. Desbois-Mouthon, A. Baron, M. J. Blivet-Van Eggelpoël et al., "Insulin-like growth factor-1 receptor inhibition induces a resistance mechanism via the epidermal growth factor receptor/HER3/AKT signaling pathway: rational basis for cotargeting insulin-like growth factor-1 receptor and epidermal growth factor receptor in hepatocellular carcinoma," Clinical Cancer Research, vol. 15, no. 17, pp. 5445-5456, 2009.

[26] M. Ariga, T. Nedachi, M. Akahori et al., "Signalling pathways of insulin-like growth factor-I that are augmented by cAMP in FRTL-5 cells," Biochemical Journal, vol. 348, no. 2, pp. 409-416, 2000.

[27] D. M. Nguyen and D. S. Schrump, "Growth factor receptors as targets for lung cancer therapy," Seminars in Thoracic and Cardiovascular Surgery, vol. 16, no. 1, pp. 3-12, 2004.

[28] K. Imai and A. Takaoka, "Comparing antibody and smallmolecule therapies for cancer," Nature Reviews Cancer, vol. 6, no. 9, pp. 714-727, 2006.

[29] M. Reck, F. Barlesi, L. Crinò et al., "Predicting and managing the risk of pulmonary haemorrhage in patients with NSCLC treated with bevacizumab: a consensus report from a panel of experts," Annals of Oncology, vol. 23, no. 5, pp. 1111-1120, 2012.

[30] P. J. Hudson and C. Souriau, "Engineered antibodies," Nature Medicine, vol. 9, no. 1, pp. 129-134, 2003.

[31] A. Gorter and S. Meri, "Immune evasion of tumor cells using membrane-bound complement regulatory proteins," Immunology Today, vol. 20, no. 12, pp. 576-582, 1999.

[32] J. Zhang, P. L. Yang, and N. S. Gray, “Targeting cancer with small molecule kinase inhibitors," Nature Reviews Cancer, vol. 9, no. 1, pp. 28-39, 2009.

[33] L. N. Johnson, "Protein kinase inhibitors: contributions from structure to clinical compounds," Quarterly Reviews of Biophysics, vol. 42, no. 1, pp. 1-40, 2009.

[34] Y. Liu and N. S. Gray, "Rational design of inhibitors that bind to inactive kinase conformations," Nature Chemical Biology, vol. 2, no. 7, pp. 358-364, 2006.

[35] P. T. C. Wan, M. J. Garnett, S. M. Roe et al., "Mechanism of activation of the RAF-ERK signaling pathway by oncogenic mutations of B-RAF," Cell, vol. 116, no. 6, pp. 855-867, 2004.

[36] E. L. Kwak, R. Sordella, D. W. Bell et al., "Irreversible inhibitors of the EGF receptor may circumvent acquired resistance to gefitinib," Proceedings of the National Academy of Sciences of the United States of America, vol. 102, no. 21, pp. 7665-7670, 2005. 
[37] A. Wissner, H. L. Fraser, C. L. Ingalls et al., "Dual irreversible kinase inhibitors: quinazoline-based inhibitors incorporating two independent reactive centers with each targeting different cysteine residues in the kinase domains of EGFR and VEGFR2," Bioorganic and Medicinal Chemistry, vol. 15, no. 11, pp. 36353648, 2007.

[38] J. E. Dancey, "Agents targeting Ras signaling pathway," Current Pharmaceutical Design, vol. 8, no. 25, pp. 2259-2267, 2002.

[39] J. H. Schiller, J. Lee, N. Hanna, A. Traynor, and D. Carbone D, "A randomized discontinuation phase II study of sorafenib versus placebo in patients with non-small cell lung cancer who have failed at least two prior chemotherapy regimens," Journal of Clinical Oncology, vol. 26, supplement 15, article 427S, 2008.

[40] G. Scagliotti, S. Novello, J. von Pawel et al., "Phase III study of carboplatin and paclitaxel alone or with sorafenib in advanced non-small-cell lung cancer," Journal of Clinical Oncology, vol. 28, no. 11, pp. 1835-1842, 2010.

[41] Y. Wang, L. Wang, Y. Liu et al., "Randomize trial of cisplatin plus gemcitabine with either sorafenib or placebo as first-line therapy for non-small cell lung cancer," Chinese Journal of Lung Cancer, vol. 14, no. 3, pp. 239-244, 2011.

[42] L. G. Paz-Ares, B. Biesma, D. Heigener et al., "Phase III, randomized, double-blind, placebo-controlled trial of gemcitabine/cisplatin alone or with sorafenib for the first-line treatment of advanced, nonsquamous non-small-cell lung cancer," Journal of Clinical Oncology, vol. 30, no. 25, pp. 3084-3092, 2012.

[43] J. S. W. Lind, A. M. C. Dingemans, H. J. M. Groen et al., "A multicenter phase II study of erlotinib and sorafenib in chemotherapy-naïve patients with advanced non-small cell lung cancer," Clinical Cancer Research, vol. 16, no. 11, pp. 30783087, 2010.

[44] C. Gridelli, F. Morgillo, A. Favaretto et al., "Sorafenib in combination with erlotinib or with gemcitabine in elderly patients with advanced non-small-cell lung cancer: a randomized phase II study," Annals of Oncology, vol. 22, no. 7, pp. 1528-1534, 2011.

[45] D. R. Spigel, H. A. Burris III, F. A. Greco et al., "Randomized, double-blind, placebo-controlled, phase II trial of sorafenib and erlotinib or erlotinib alone in previously treated advanced nonsmall-cell lung cancer," Journal of Clinical Oncology, vol. 29, no. 18, pp. 2582-2589, 2011.

[46] A. Adjei, G. Blumenschein Jr., S. Mandrekar, S. Hillman, U. Gatzemeier, and D. Heigener, "Long-term safety and tolerability of sorafenib in patients with advanced non-small-cell lung cancer: a case-based review," Clinical Lung Cancer, vol. 12, no. 4, pp. 212-217, 2011.

[47] D. B. Mendel, A. Douglas Laird, X. Xin et al., "In vivo antitumor activity of SU11248, a novel tyrosine kinase inhibitor targeting vascular endothelial growth factor and plateletderived growth factor receptors: determination of a pharmacokinetic/pharmacodynamic relationship," Clinical Cancer Research, vol. 9, no. 1, pp. 327-337, 2003.

[48] S. Novello, G. V. Scagliotti, R. Rosell et al., "Phase II study of continuous daily sunitinib dosing in patients with previously treated advanced non-small cell lung cancer," British Journal of Cancer, vol. 101, no. 9, pp. 1543-1548, 2009.

[49] S. Novello, C. Camps, F. Grossi et al., "Phase II study of sunitinib in patients with non-small cell lung cancer and irradiated brain metastases," Journal of Thoracic Oncology, vol. 6, no. 7, pp. 12601266, 2011.

[50] G. V. Scagliotti, I. Vynnychenko, K. Park et al., "International, randomized, placebo-controlled, double-blind phase III study of motesanib plus carboplatin/paclitaxel in patients with advanced nonsquamous non-small-cell lung cancer: MONET1," Journal of Clinical Oncology, vol. 30, no. 23, pp. 2829-2836, 2012.

[51] M. A. Socinski, F. A. Scappaticci, M. Samant, M. M. Kolb, and M. F. Kozloff, "Safety and efficacy of combining sunitinib with bevacizumab + paclitaxel/carboplatin in non-small cell lung cancer," Journal of Thoracic Oncology, vol. 5, no. 3, pp. 354-360, 2010.

[52] G. R. Blumenschein Jr., T. Ciuleanu, F. Robert et al., "Sunitinib plus erlotinib for the treatment of advanced/metastatic nonsmall-cell lung cancer: a lead-in study," Journal of Thoracic Oncology, vol. 7, no. 9, pp. 1406-1416, 2012.

[53] P. Nikolinakos and J. V. Heymach, "The tyrosine kinase inhibitor cediranib for non-small cell lung cancer and other thoracic malignancies," Journal of Thoracic Oncology, vol. 3, no. 6, pp. S131-S134, 2008.

[54] N. Yamamoto, T. Tamura, N. Yamamoto et al., "Phase I, dose escalation and pharmacokinetic study of cediranib (RECENTINU゚), a highly potent and selective VEGFR signaling inhibitor, in Japanese patients with advanced solid tumors," Cancer Chemotherapy and Pharmacology, vol. 64, no. 6, supplement 2, pp. 1165-1172, 2009.

[55] G. Goss, F. A. Shepherd, S. Laurie et al., "A phase I and pharmacokinetic study of daily oral cediranib, an inhibitor of vascular endothelial growth factor tyrosine kinases, in combination with cisplatin and gemcitabine in patients with advanced non-small cell lung cancer: a study of the National Cancer Institute of Canada Clinical Trials Group," European Journal of Cancer, vol. 45, no. 5, pp. 782-788, 2009.

[56] G. D. Goss, A. Arnold, F. A. Shepherd et al., "Randomized, double-blind trial of carboplatin and paclitaxel with either daily oral cediranib or placebo in advanced non-small-cell lung cancer: NCIC clinical trials group BR24 study," Journal of Clinical Oncology, vol. 28, no. 1, pp. 49-55, 2010.

[57] G. K. Dy, S. J. Mandrekar, G. D. Nelson et al., "A randomized phase II study of gemcitabine and carboplatin with or without cediranib as first-line therapy in advanced non-small-cell lung cancer: North Central Cancer Treatment Group Study N0528," Journal of Thoracic Oncology, vol. 8, no. 1, pp. 79-88, 2013.

[58] D. H. Albert, P. Tapang, T. J. Magoc et al., "Preclinical activity of ABT-869, a multitargeted receptor tyrosine kinase inhibitor," Molecular Cancer Therapeutics, vol. 5, no. 4, pp. 995-1006, 2006.

[59] H. Asahina, Y. Tamura, H. Nokihara et al., "An open-label, phase 1 study evaluating safety, tolerability, and pharmacokinetics of linifanib (ABT-869) in Japanese patients with solid tumors," Cancer Chemotherapy and Pharmacology, vol. 69, no. 6, pp. 1477-1486, 2012.

[60] E. H. Tan, G. D. Goss, R. Salgia et al., "Phase 2 trial of linifanib (ABT-869) in patients with advanced non-small cell lung cancer," Journal of Thoracic Oncology, vol. 6, no. 8, pp. 14181425, 2011.

[61] T. K. Choueiri, "Axitinib, a novel anti-angiogenic drug with promising activity in various solid tumors," Current Opinion in Investigational Drugs, vol. 9, no. 6, pp. 658-671, 2008.

[62] F. Wang, Y. J. Mi, S. G. Chen et al., "Axitinib targeted cancer stemlike cells to enhance efficacy of chemotherapeutic drugs via inhibiting the drug transport function of ABCG2," Molecular Medicine, vol. 18, pp. 887-898, 2012.

[63] M. F. Kozloff, L. P. Martin, M. Krzakowski et al., "Phase I trial of axitinib combined with platinum doublets in patients with advanced non-small cell lung cancer and other solid tumours," British Journal of Cancer, vol. 107, no. 8, pp. 1277-1285, 2012. 
[64] K. P. S. Raghav and G. R. Blumenschein, "Motesanib and advanced NSCLC: experiences and expectations," Expert Opinion on Investigational Drugs, vol. 20, no. 6, pp. 859-869, 2011.

[65] A. Coxon, B. Ziegler, S. Kaufman et al., "Antitumor activity of motesanib alone and in combination with cisplatin or docetaxel in multiple human non-small-cell lung cancer xenograft models," Molecular Cancer, vol. 11, p. 70, 2012.

[66] M. Das and H. Wakelee, "Anti-angiogenic agents in Non-SmallCell Lung Cancer (NSCLC): a perspective on the MONET1 (Motesanib NSCLC Efficacy and Tolerability) study," Journal of Thoracic Disease, vol. 4, no. 6, pp. 558-561, 2012.

[67] S. R. Wedge, D. J. Ogilvie, M. Dukes et al., "ZD6474 inhibits vascular endothelial growth factor signaling, angiogenesis, and tumor growth following oral administration," Cancer Research, vol. 62, no. 16, pp. 4645-4655, 2002.

[68] L. Zhang, S. Li, Y. Zhang et al., "Pharmacokinetics and tolerability of vandetanib in Chinese patients with solid, malignant tumors: an open-label, phase I, rising multiple-dose study," Clinical Therapeutics, vol. 33, no. 3, pp. 315-327, 2011.

[69] J. V. Heymach, L. Paz-Ares, F. De Braud et al., "Randomized phase II study of vandetanib alone or with paclitaxel and carboplatin as first-line treatment for advanced non-small-cell lung cancer," Journal of Clinical Oncology, vol. 26, no. 33, pp. 5407-5415, 2008.

[70] R. H. De Boer, Ó. Arrieta, C. H. Yang et al., "Vandetanib plus pemetrexed for the second-line treatment of advanced nonsmall-cell lung cancer: a randomized, double-blind phase III trial," Journal of Clinical Oncology, vol. 29, no. 8, pp. 1067-1074, 2011.

[71] R. S. Herbst, Y. Sun, W. E. E. Eberhardt et al., "Vandetanib plus docetaxel versus docetaxel as second-line treatment for patients with advanced non-small-cell lung cancer (ZODIAC): a doubleblind, randomised, phase 3 trial," The Lancet Oncology, vol. 11, no. 7, pp. 619-626, 2010.

[72] J. S. Lee, V. Hirsh, K. Park et al., "Vandetanib Versus placebo in patients with advanced non-small-cell lung cancer after prior therapy with an epidermal growth factor receptor tyrosine kinase inhibitor: a randomized, double-blind phase III trial (ZEPHYR)," Journal of Clinical Oncology, vol. 30, no. 10, pp. 1114-1121, 2012.

[73] H. A. Yu and G. J. Riely, "Second-generation epidermal growth factor receptor tyrosine kinase inhibitors in lung cancers," Journal of the National Comprehensive Cancer Network, vol. 11, no. 2, pp. 161-169, 2013.

[74] M. C. Pietanza, S. M. Gadgeel, A. Dowlati et al., "Phase II study of the multitargeted tyrosine kinase inhibitor XL647 in patients with non-small-cell lung cancer," Journal of Thoracic Oncology, vol. 7, no. 5, pp. 856-865, 2012.

[75] S. B. Gendreau, R. Ventura, P. Keast et al., "Inhibition of the T790M gatekeeper mutant of the epidermal growth factor receptor by EXEL-7647," Clinical Cancer Research, vol. 13, no. 12, pp. 3713-3723, 2007.

[76] M. C. Pietanza, T. J. Lynch Jr., P. N. Lara et al., "XL647-A multitargeted tyrosine kinase inhibitor: results of a phase II study in subjects with non-small cell lung cancer who have progressed after responding to treatment with either gefitinib or erlotinib," Journal of Thoracic Oncology, vol. 7, no. 1, pp. 219226, 2012.

[77] P. H. Marathe, A. V. Kamath, Y. Zhang, C. D’Arienzo, R. Bhide, and J. Fargnoli, "Preclinical pharmacokinetics and in vitro metabolism of brivanib (BMS-540215), a potent VEGFR2 inhibitor and its alanine ester prodrug brivanib alaninate,"
Cancer Chemotherapy and Pharmacology, vol. 65, no. 1, pp. 5566, 2009.

[78] R. S. Bhide, Z. W. Cai, Y. Z. Zhang et al., "Discovery and preclinical studies of (R)-1-(4-(4-fluoro-2-methyl-1H-indol-5-yloxy)-5methylpyrrolo[2,1-f] $[1,2,4]$ triazin-6-yloxy)propan-2-ol (BMS$540215)$, an in vivo active potent VEGFR-2 inhibitor," Journal of Medicinal Chemistry, vol. 49, no. 7, pp. 2143-2146, 2006.

[79] R. S. Bhide, L. J. Lombardo, J. T. Hunt et al., "The antiangiogenic activity in xenograft models of brivanib, a dual inhibitor of vascular endothelial growth factor receptor-2 and fibroblast growth factor receptor-1 kinases," Molecular Cancer Therapeutics, vol. 9, no. 2, pp. 369-378, 2010.

[80] T. Mekhail, E. Masson, B. S. Fischer et al., "Metabolism, excretion, and pharmacokinetics of oral brivanib in patients with advanced or metastatic solid tumors," Drug Metabolism and Disposition, vol. 38, no. 11, pp. 1962-1966, 2010.

[81] D. J. Jonker, L. S. Rosen, M. B. Sawyer et al., "A phase I study to determine the safety, pharmacokinetics and pharmacodynamics of a dual VEGFR and FGFR inhibitor, brivanib, in patients with advanced or metastatic solid tumors," Annals of Oncology, vol. 22, no. 6, pp. 1413-1419, 2011.

[82] F. Lee, M. N. Jure-Kunkel, and M. E. Salvati, "Synergistic activity of ixabepilone plus other anticancer agents: preclinical and clinical evidence," Therapeutic Advances in Medical Oncology, vol. 3, no. 1, pp. 11-25, 2011.

[83] F. Hilberg, G. J. Roth, M. Krssak et al., "BIBF 1120: triple angiokinase inhibitor with sustained receptor blockade and good antitumor efficacy," Cancer Research, vol. 68, no. 12, pp. 4774-4782, 2008.

[84] M. Reck, R. Kaiser, C. Eschbach et al., "A phase II doubleblind study to investigate efficacy and safety of two doses of the triple angiokinase inhibitor BIBF 1120 in patients with relapsed advanced non-small-cell lung cancer," Annals of Oncology, vol. 22, no. 6, pp. 1374-1381, 2011.

[85] P. M. Ellis, R. Kaiser, Y. Zhao, P. Stopfer, S. Gyorffy, and N. Hanna, "Phase I open-label study of continuous treatment with BIBF 1120, a triple angiokinase inhibitor, and pemetrexed in pretreated non-small cell lung cancer patients," Clinical Cancer Research, vol. 16, no. 10, pp. 2881-2889, 2010.

[86] R. C. Doebele, P. Conkling, A. M. Traynor et al., "A phase I, open-label dose-escalation study of continuous treatment with BIBF, 1120 in combination with paclitaxel and carboplatin as first-line treatment in patients with advanced non-small-cell lung cancer," Annals of Oncology, vol. 23, no. 8, pp. 2094-2102, 2012.

[87] R. Kumar, V. B. Knick, S. K. Rudolph et al., "Pharmacokineticpharmacodynamic correlation from mouse to human with pazopanib, a multikinase angiogenesis inhibitor with potent antitumor and antiangiogenic activity," Molecular Cancer Therapeutics, vol. 6, no. 7, pp. 2012-2021, 2007.

[88] N. Altorki, M. E. Lane, T. Bauer et al., "Phase II proof-of-concept study of pazopanib monotherapy in treatment-naive patients with stage I/II resectable non-small-cell lung cancer," Journal of Clinical Oncology, vol. 28, no. 19, pp. 3131-3137, 2010.

[89] R. Plummer, A. Madi, M. Jeffels et al., "A Phase I study of pazopanib in combination with gemcitabine in patients with advanced solid tumors," Cancer Chemotherapy and Pharmacology, vol. 71, no. 1, pp. 93-101, 2013.

[90] C. P. Belani, G. Goss, and G. Blumenschein, "Recent clinical developments and rationale for combining targeted agents in non-small cell lung cancer (NSCLC)," Cancer Treatment Reviews, vol. 38, no. 3, pp. 173-184, 2012. 
[91] Y. L. Choi, M. Soda, Y. Yamashita et al., "EML4-ALK mutations in lung cancer that confer resistance to ALK inhibitors," New England Journal of Medicine, vol. 363, no. 18, pp. 1734-1739, 2010.

[92] C. J. Langer, T. Mok, and P. E. Postmus, “Targeted agents in the third-/fourth-line treatment of patients with advanced (stage III/IV) non-small cell lung cancer (NSCLC)," Cancer Treatment Reviews, vol. 39, no. 3, pp. 252-260, 2013.

[93] E. S. Kim, R. S. Herbst, I. I. Wistuba et al., "The BATTLE trial: personalizing therapy for lung cancer," Cancer Discovery, vol. 1, no. 1, pp. 44-53.

[94] T. S. Mok, Y. L. Wu, S. Thongprasert et al., "Gefitinib or carboplatin-paclitaxel in pulmonary adenocarcinoma," New England Journal of Medicine, vol. 361, no. 10, pp. 947-957, 2009.

[95] M. Ladanyi and W. Pao, "Lung adenocarcinoma: guiding EGFR-targeted therapy and beyond," Modern Pathology, vol. 21, supplement 2, pp. S16-S22, 2008.

[96] V. A. Miller, M. G. Kris, N. Shah et al., "Bronchioloalveolar pathologic subtype and smoking history predict sensitivity to gefitinib in advanced non-small-cell lung cancer," Journal of Clinical Oncology, vol. 22, no. 6, pp. 1103-1109, 2004.

[97] G. Holgersson, S. Bergström, M. Bergqvist et al., "Swedish Lung Cancer Radiation Study Group: pedictive value of histology for radiotherapy response in patients with non-small cell lung cancer," European Journal of Cancer, vol. 47, no. 16, pp. 24152421, 2011.

[98] J. A. Engelman, K. Zejnullahu, T. Mitsudomi et al., "MET amplification leads to gefitinib resistance in lung cancer by activating ERBB3 signaling," Science, vol. 316, no. 5827, pp. 10391043, 2007. 


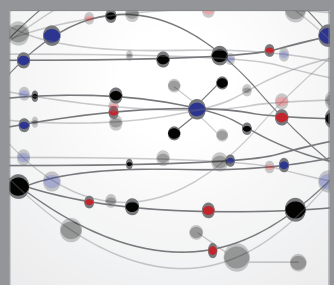

The Scientific World Journal
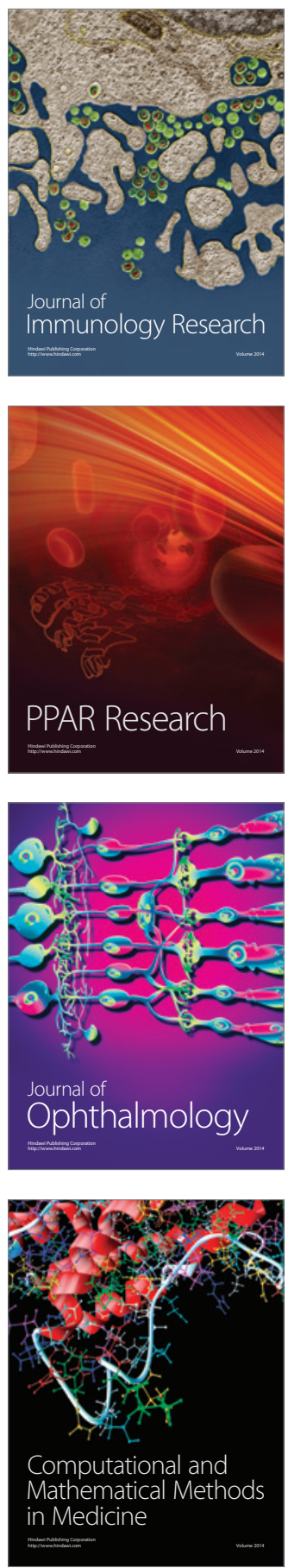

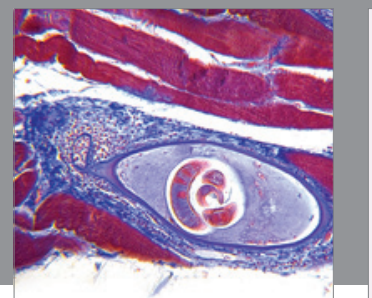

Gastroenterology

Research and Practice
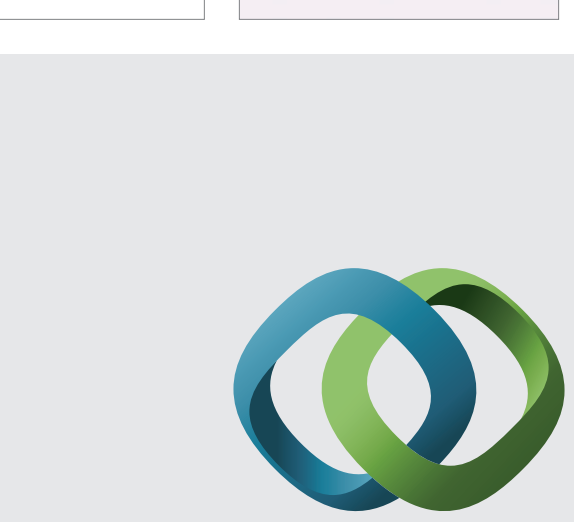

\section{Hindawi}

Submit your manuscripts at

http://www.hindawi.com
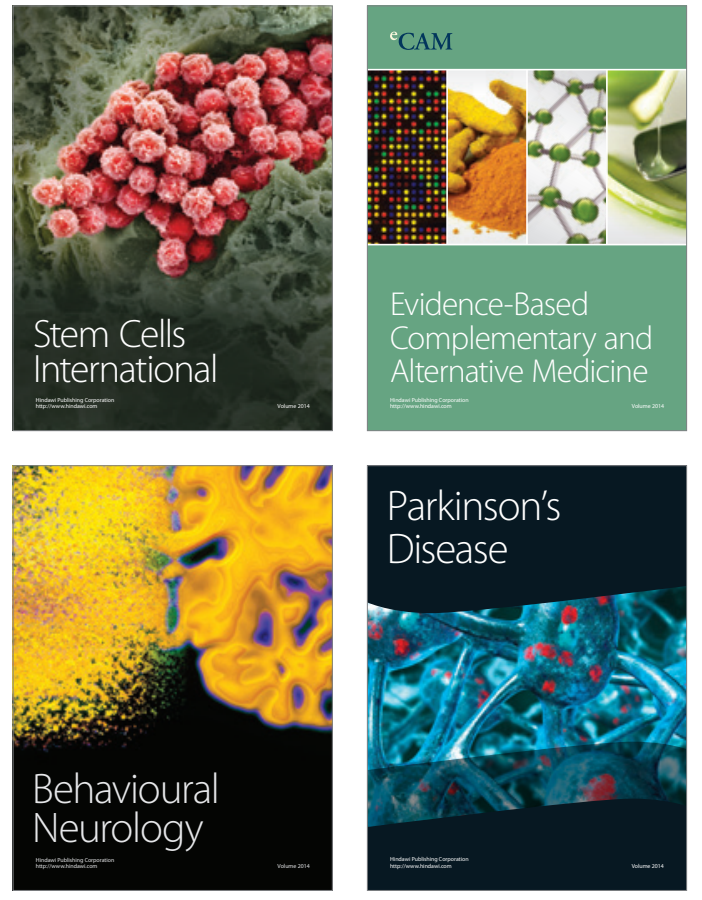
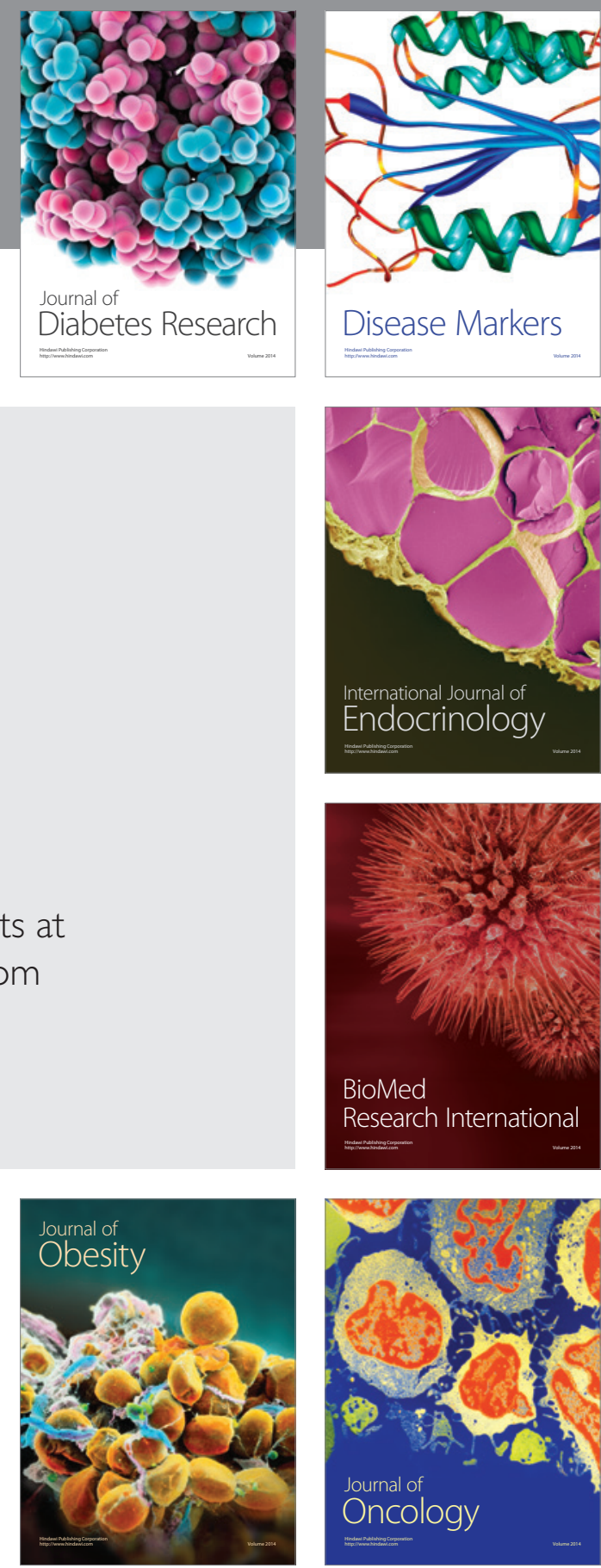

Disease Markers
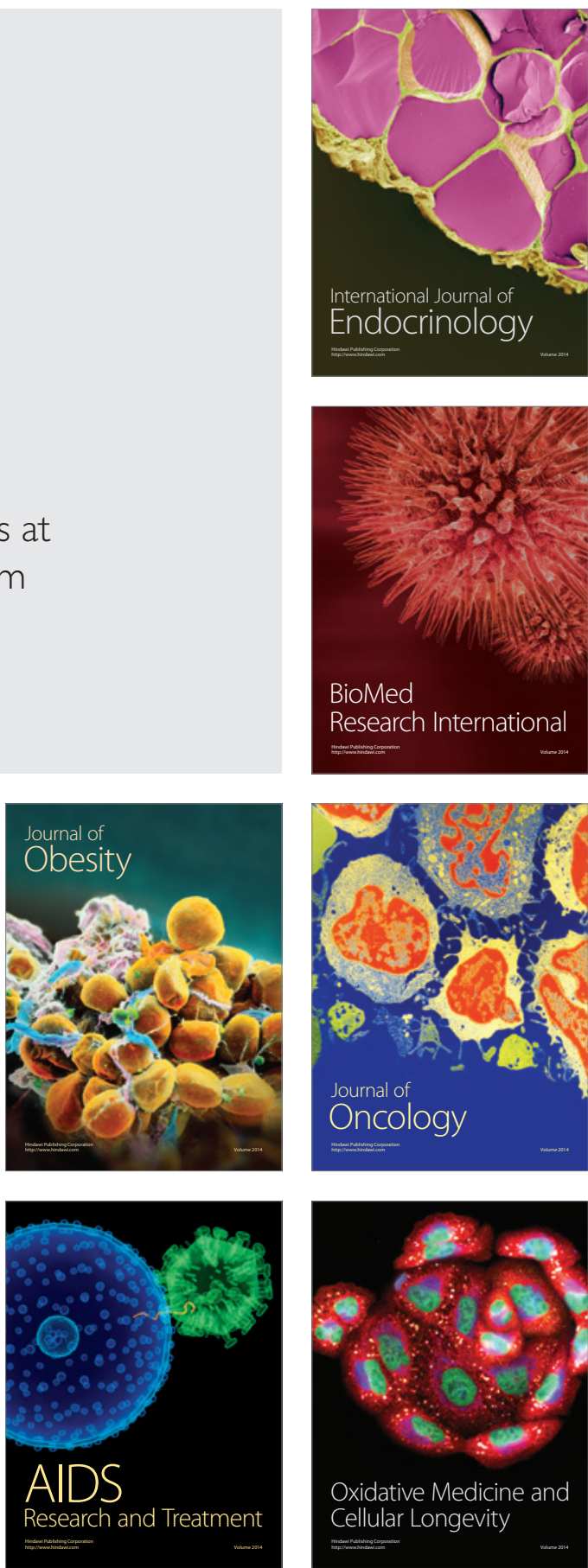